\title{
LITERATURBERICHT
}

Johanna Ray Vollhardt/J. Christopher Cohrs

\section{Aktuelle sozialpsychologische Beiträge zur Friedens- und Konfliktforschung}

Dieser Artikel gibt einen Überblick über die aktuelle sozialpsychologische Forschung im Hinblick auf Themen, die für die Friedens- und Konfliktforschung relevant sind. Auf Grundlage der Ergebnisse einer inhaltsanalytischen Untersuchung sozialpsychologischer Friedensforschung (Vollhardt/Bilali 2008) besprechen wir Artikel, die im Zeitraum 2008 bis 2012 in Zeitschriften publiziert wurden, welche die Mainstream-Sozialpsychologie repräsentieren: "Journal of Personality and Social Psychology«, »Personality and Social Psychology Bulletin«, »European Journal of Social Psychology«, und »Basic and Applied Social Psychology«. Aktuelle Trends, die für die Friedens- und Konfliktforschung relevant sind, werden vorgestellt und kritisch diskutiert. Wir diskutieren außerdem, wie das Potenzial sozialpsychologischer Forschung, zur interdisziplinären Friedens- und Konfliktforschung beizutragen, mit Hilfe der Befreiungspsychologie und anderer, kritischerer Strömungen innerhalb der Psychologie erhöht werden kann. Dies umfasst eine stärkere Internationalisierung der Disziplin, mehr Feldforschung, Zusammenarbeit mit NGOs, und eine stärkere Kontextualisierung von Forschungsfragen sowie Berücksichtigung von Einflüssen auf der Makroebene. Schließlich stellen wir auch kurz die sozialpsychologische Friedensforschung und entsprechende Organisationsstrukturen in Deutschland vor.

Schlagworte: Sozialpsychologie, Friedenspsychologie, sozialpsychologische Friedensforschung, kontextuelle Psychologie, Befreiungspsychologie

\section{Aktuelle Sozialpsychologische Beiträge zur Friedens- und Konfliktforschung}

Seit ihrem Beginn hat sich die Sozialpsychologie intensiv mit Frieden und Konflikt auseinandergesetzt (z. B. Kelman 2012). Einige der klassischen sozialpsychologischen Untersuchungen, wie z. B. die Milgram-Experimente des Psychologen Stan- 
ley Milgram oder Muzafer Sherifs Robbers-Cave-Studien, beziehen sich unmittelbar auf Gewalt sowie Konfliktentstehung und -lösung. Wie sieht das heute aus? Was kann die aktuelle empirische Sozialpsychologie zur interdisziplinären Friedens- und Konfliktforschung beisteuern? Dieser Beitrag hat das Ziel, in dieser Hinsicht einen Überblick über die derzeitige Lage der (Mainstream-)Sozialpsychologie zu bieten und kritisch zu diskutieren.

Im Folgenden werden wir zunächst Definitionen der Sozialpsychologie und der Friedenspsychologie geben sowie einen Schwerpunkt auf die sozialpsychologische Friedensforschung setzen (in Abgrenzung von anderen Teildisziplinen der Psychologie, die auch zur Friedenspsychologie beitragen). In einem nächsten Schritt werden wir eine inhaltsanalytische Untersuchung zusammenfassend diskutieren, die einen ersten systematischen Überblick über die sozialpsychologische Friedensforschung bietet. Daran anschließend wird eine thematische Analyse der letzten fünf Jahrgänge (2008-2012) derjenigen Zeitschriften, die in dieser Inhaltsanalyse ausgewertet wurden, vorgestellt. Anhand dessen wird diskutiert, welche Trends seitdem in der sozialpsychologischen Friedensforschung, wie sie in Mainstream-Zeitschriften vertreten ist, zu beobachten sind. Da sich dieser Beitrag an ein deutschsprachiges Publikum richtet, stellen wir danach kurz die friedenspsychologische Forschung in Deutschland vor, in der eine sozialpsychologische Ausrichtung repräsentiert ist. Wir diskutieren abschließend, was die sozialpsychologische Friedensforschung der interdisziplinären Friedens- und Konfliktforschung bieten kann und wie beide Bereiche ihren Wirkungsgrad erweitern können, indem sie sich um eine Integration von Perspektiven bemühen.

\section{Sozialpsychologie, Friedenspsychologie und sozialpsychologische Friedensforschung}

Sozialpsychologie ist ein Teilgebiet der Psychologie, das sich damit beschäftigt, wie das individuelle Denken, Fühlen und Handeln durch die tatsächliche, imaginierte oder implizierte Anwesenheit anderer beeinflusst wird (Allport 1954). Dies ist zumindest eine weit verbreitete Lehrbuchdefinition, die sich in der individualistisch ausgerichteten, empirisch orientierten, westlich geprägten Sozialpsychologie der letzten Jahrzehnte, wie sie auch an Universitäten gelehrt wird, durchgesetzt hat (Greenwood 2004) - trotz zeitweise starker Kritik (z. B. Gergen 1973; 2006). Gegenwärtig weniger verbreitete Definitionen der Sozialpsychologie, die den Ursprüngen dieser Disziplin sowie der Friedens- und Konfliktforschung näherstehen, beziehen sich stärker auf die Interaktion zwischen Individuen und sozialen Strukturen. So kann die Sozialpsychologie beispielsweise auch als eine Disziplin be- 
zeichnet werden, die sich mit der Schnittstelle zwischen individuellem Handeln und gesellschaftlich-institutionellen Prozessen beschäftigt (Kelman 1965; 2012). Es ist dieser Auffassung nach nicht möglich, individuelles Handeln zu verstehen, ohne zu berücksichtigen, wie der soziale (einschließlich institutionelle) Kontext und individualpsychologische Prozesse zusammenwirken (Pettigrew 2011). Diese Variante der Sozialpsychologie kann mit Thomas F. Pettigrew (1991) als »kontextuelle Sozialpsychologie« bezeichnet werden. Sie steht im starken Kontrast zur MainstreamSozialpsychologie, die häufig de-kontextualisiert ist und sich auf isolierte, individualpsychologische, mentale Prozesse konzentriert, die überwiegend in experimenteller Laborforschung untersucht werden (für kritische Diskussionen vgl. Bar-Tal 2004; Greenwood 2004; Kelman 2012; Pettigrew 2011).

$\mathrm{Zu}$ den übergeordneten, allgemeinen Themenbereichen der Sozialpsychologie zählen z. B. Einstellungen und Persuasion, Konformität und Gehorsam, Vorurteile und Stereotype, Gruppendynamik, Selbst und Identität, Aggression, prosoziales Handeln, interpersonelle Wahrnehmung und interpersonelle Attraktion (Aronson et al. 2013; Bierhoff 2006). Die Relevanz vieler dieser Themen für die Friedens- und Konfliktforschung liegt auf der Hand und wird im Folgenden näher diskutiert.

Die Friedenspsychologie ist weniger etabliert als die Sozialpsychologie und wurde erst Mitte der 1980er Jahre als Teilbereich der Psychologie bezeichnet (Christie et al. 2001). Ihre Wurzeln sind jedoch viel älter, und das Interesse an dem Gegenstandsbereich bestand lange bevor dieser institutionalisiert wurde (Christie et al. 2008). Morton Deutsch (1995) z. B. bezeichnete William James, der oft als »Vater« der Psychologie angesehen wird, als den ersten Friedenspsychologen; wegen seines Aufsatzes »The Moral Equivalent of War «, in dem er psychologische Alternativen zum Krieg diskutierte (James 1995). In Reaktion auf den Ersten und Zweiten Weltkrieg sowie später den Kalten Krieg gab es in der Geschichte der Psychologie zahlreiche Forschungsfragen, die heutzutage als Friedenspsychologie angesehen werden könnten. Viele davon fallen in den Bereich der Sozialpsychologie (Gibson 2011; Kelman 2012). Beispielsweise erhielt die Einstellungsforschung einen wichtigen Antrieb durch das Interesse an der Entwicklung von Propaganda, die sich an demoralisierte amerikanische Soldaten während des Zweiten Weltkriegs richtete (Hovland et al. 1953; Aronson et al. 2013); und Milgrams berühmte GehorsamExperimente waren von den Eichmann-Prozessen und der Rolle des Gehorsams während des Holocausts angeregt (Milgram 1974). Der Einfluss nationaler wie internationaler Konflikte (z. B. auch die ethnischen Konflikte der 1990er Jahre, die Terroranschläge am 11. September 2001 und die darauf folgenden Kriege) auf die Themen, die in der (friedenspsychologischen) Sozialpsychologie besprochen und 
untersucht werden, setzt sich bis heute fort (Christie 2006; Christie et al. 2008; Gibson 2011).

Die Friedenspsychologie ist streng genommen keine eigene Teildisziplin und ist nicht mit der Sozialpsychologie gleichzusetzen. Es gibt allerdings Überlappungen zwischen diesen beiden Bereichen und sozialpsychologische Forschung spielt innerhalb der Friedenspsychologie eine wichtige Rolle (Boehnke et al. 2005; Christie 2006; Cohrs/Boehnke 2008; Kelman 2012; Vollhardt/Bilali 2008). Die Friedenspsychologie hat aber ihren eigenen Gegenstandsbereich und sie ist ihrem Selbstverständnis nach normativ ausgerichtet. Sie fokussiert, in Anlehnung an Johan Galtungs (1969) Unterscheidung zwischen positivem und negativem Frieden, auf psychologische Theorien und Praktiken zur Verhinderung und Reduktion direkter und struktureller Gewalt sowie zur (möglichst) gewaltfreien Lösung von Konflikten und der Schaffung nachhaltigen Friedens durch soziale Gerechtigkeit (Christie et al. 2001). Um diese komplexen Ziele zu erreichen, greift die Friedenspsychologie auf die theoretischen und methodologischen Bausteine unterschiedlicher Teildisziplinen der Psychologie zurück. Die Friedenspsychologie ist aber auch notwendigerweise interdisziplinärer ausgerichtet und legt mehr Wert auf gesellschaftliche, strukturelle Einflüsse als viele andere Teildisziplinen der Psychologie (Christie 2006; Kelman 2012; Pettigrew 2011). Friedenspsychologische Organisationen, Tagungen und Publikationen sind außerdem insgesamt relativ international ausgerichtet (z. B. Christie et al. 2001). Da sich friedenspsychologische Forschung aber mit aktuellen geopolitischen Konflikten sowie positivem und negativem Frieden im jeweiligen gesellschaftlichen Kontext beschäftigt, gibt es auch regionale Schwerpunktsetzungen innerhalb der Friedenspsychologie (Christie 2006). ${ }^{1}$

Als »sozialpsychologische Friedensforschung « bezeichnen wir einen überlappenden Forschungsbereich, der gleichermaßen als Sozialpsychologie oder als Friedenspsychologie eingeordnet werden könnte (Cohrs/Boehnke 2008; Gibson 2011; Kelman 2012; Vollhardt/Bilali 2008). Sozialpsychologische Friedensforschung befasst sich mit Kognitionen (z. B. Einstellungen, Vorurteilen, Ideologien), Emotionen (z. B. Zorn, Schuld) und Handlungen (z. B. Diskriminierung, prosoziales Verhalten), die zu direkter und struktureller Gewalt sowie ihrer Verhinderung und Reduktion oder zur (möglichst) gewaltfreien Lösung von Konflikten und der Schaffung nachhaltigen Friedens beitragen. Die aktuelle sozialpsychologische (westlich orientierte) Friedenspsychologie ist stark auf Intergruppenbeziehungen konzentriert

1 Über Friedenspsychologie in Deutschland siehe z. B. Boehnke et al. (2005); in Australien: Bretherton/Balvin (2012); in Lateinamerika: Burton/Kagan (2005); in Afrika: Heuchert (2012); in Asien: Montiel/Noor (2009). 
(Vollhardt/Bilali 2008). Einige kritische Stimmen merken an, dass dadurch strukturelle Faktoren wie Staat und Militär zu sehr vernachlässigt werden (Gibson 2011), und wieder andere, dass interpersoneller Frieden sowie »innerer Frieden « mehr beachtet werden sollten (siehe hierfür die Diskussion in Christie et al. 2008). In Abgrenzung zur entwicklungspsychologisch ausgerichteten Friedensforschung (Barber 2009; Daiute 2010; Hammack 2010) setzt sich die sozialpsychologische Friedensforschung nicht spezifisch mit Prozessen bei Kindern und Jugendlichen auseinander; in Abgrenzung zur klinisch ausgerichteten Friedenspsychologie befasst sie sich nicht mit Kriegstrauma und Resilienz oder mit Phänomenen wie psychologischen Störungen und Anpassungen bei KindersoldatInnen (Kaminer et al. 2013; Wessells 2006). Es gilt jedoch zu beachten, dass sich nicht alle SozialpsychologInnen, die sozialpsychologische Friedensforschung betreiben, als FriedenspsychologInnen identifizieren würden (Cohrs/Boehnke 2008; Kelman 2012; Vollhardt/Bilali 2008). Dies hängt vielleicht mit einem gewissen Stigma zusammen, das der Friedenspsychologie anhaftet - wie z. B. dass für manche der Begriff »Frieden« unwissenschaftlich klingt sowie »weich, schwach, naiv, idealistisch und sogar gefährlich und unpatriotisch « (Christie et al. 2008: 548; eigene Übersetzung), oder damit, dass die Sozialpsychologie etablierter ist und einen höheren Status in Bezug auf Institutionalisierung und Verfügbarkeit von Ressourcen wie Stellen, Forschungsgelder oder Zeitschriften besitzt. Um sich einen Überblick über das Ausmaß und die Themen der sozialpsychologischen Friedensforschung im Mainstream zu verschaffen, ist es also notwendig, auch relevante Publikationen mit einzubeziehen, die das Wort »Frieden« nicht im Titel tragen oder als Schlüsselbegriff verwenden.

\section{Merkmale der sozialpsychologischen Friedensforschung}

Im Folgenden stellen wir einige Merkmale der sozialpsychologischen Friedensforschung genauer vor. Dazu greifen wir auf eine inhaltsanalytische Untersuchung entsprechender Publikationen in einer Auswahl relevanter Zeitschriften zurück.

\subsection{Inhaltsanalytische Untersuchung zur sozialpsychologischen Friedensforschung: Methode}

Ein erster Versuch, die Beiträge der Mainstream-Sozialpsychologie zur Friedenforschung systematisch zu untersuchen, wurde von Johanna Ray Vollhardt und Rezarta Bilali (2008) unternommen. Dieser Beitrag erschien in einem von J. Christopher Cohrs und Klaus Boehnke herausgegebenen Sonderheft zu »Social Psychology and Peace« in der deutschen Zeitschrift »Social Psychology« (ehemals »Zeitschrift für Sozialpsychologie«). Die Autorinnen berichteten die Ergebnisse einer inhalts- 
analytischen Auswertung von fünf Jahrgängen (2001-2005) aus vier allgemeinen sozialpsychologischen Zeitschriften - »Basic and Applied Social Psychology" (BASP), »European Journal of Social Psychology « (EJSP), »Journal of Personality and Social Psychology « (JPSP) und »Personality and Social Psychology Bulletin« (PSPB) - sowie einer überwiegend sozialpsychologisch ausgerichteten Zeitschrift - »Journal of Social Issues « (JSI) -, die sich auf soziale Probleme spezialisiert. Dies sollte eine relativ repräsentative Auswahl an Mainstream-Publikationen in der Sozialpsychologie darstellen. Für die Auswahl wurden Zeitschriftenrankings, geographische Verortung sowie die Balance zwischen grundlagen- und anwendungsorientierten Arbeiten in Betracht gezogen. So sind drei der ausgewählten Zeitschriften (JPSP, PSPB und EJSP) hoch im Ranking innerhalb der Disziplin, während die anderen beiden (BASP und JSI) im Mittelfeld verortet sind. Mit dem EJSP ist eine Zeitschrift dabei, die insbesondere die europäische (und nicht nur die amerikanische) Sozialpsychologie vertritt. Zwei der ausgesuchten Zeitschriften (BASP und JSI) sind außerdem anwendungsorientierter. Zusätzlich wurde eine zentrale friedenspsychologische Zeitschrift (»Peace and Conflict: Journal of Peace Psychology«) analysiert. Das Ziel dieser Inhaltsanalyse war, herauszufinden, welcher Anteil publizierter sozialpsychologischer Forschung in diesen Zeitschriften sich als sozialpsychologische Friedensforschung qualifiziert und, umgekehrt, welcher Anteil explizit friedenspsychologischer Publikationen sich auf sozialpsychologische Theorien und Modelle stützt. Es sollte also der Überlappungsbereich von Sozialund Friedenspsychologie genauer beschrieben werden.

Die Auswahl und Kodierung relevanter Artikel in diesen Zeitschriften erfolgte anhand von vier Kriterien der sozialpsychologischen Friedenspsychologie, die unter Rückgriff auf die einschlägige friedenspsychologische und sozialpsychologische Literatur (z. B. Christie et al. 2001; Christie 2006; Fuchs/Sommer 2004; Pettigrew $1998,2006)$ eigens für diese Untersuchung entwickelt wurden. Neben der thematischen Relevanz (im Hinblick auf Gewalt und Konflikt sowie positiven und negativen Frieden zwischen sozialen Gruppen) wurde außerdem untersucht, ob die in den Artikeln berichtete Forschung 1) normativ (statt wertfrei) ist, und zwar mit dem Ziel der Reduktion und Verhinderung von Konflikt und Gewalt und/oder der Herbeiführung positiven oder negativen Friedens; 2) kontextualisiert, d. h. die Forschungsfragen in ihren relevanten sozialen Kontext einbettet, anstatt davon zu abstrahieren; 3) mehrere Analyseebenen einbezieht, und zwar entweder empirisch (z. B. durch Mehrebenenanalyse) oder zumindest in der theoretischen Modellbildung; und 4) durch eine praktische anstelle einer rein epistemischen Orientierung der Forschungsfragen (Carrier 2004) versucht, die Welt nicht nur zu verstehen, sondern auch zu verändern (Martín-Baró 1994). Obwohl diese Kriterien nicht der 
derzeitigen prototypischen Sozialpsychologie entsprechen, sind diese Ausrichtungen doch auch in der Disziplin vorhanden und verankert. Einige kritische Stimmen würden diese Merkmale sogar als eine originalgetreuere Variante der (kontextuellen) Sozialpsychologie ansehen (Bar-Tal 2004; Greenwood 2004; Kelman 2012; Pettigrew 1991; 1998).

\subsection{Ergebnisse der Inhaltsanalyse}

Anhand dieser Kriterien wurden alle Artikel analysiert, die in einem Zeitraum von fünf Jahren (2001-2005) in den ausgewählten Zeitschriften veröffentlicht wurden $(N=2125)$. Artikel wurden als friedenspsychologische Sozialpsychologie klassifiziert, wenn die Forschungsfragen a) für Frieden und Konflikt auf der gesellschaftlichen oder Intergruppen-Ebene relevant waren; b) sozialpsychologische Theorien, Modelle oder Konstrukte verwendet wurden und c) mindestens drei der vier oben genannten Kriterien erfüllt waren. Von den 2125 Artikeln wurden 205 als sozialpsychologische Friedenspsychologie eingeordnet. 170 davon wurden in den sozialpsychologischen Zeitschriften veröffentlicht, was $8 \%$ aller publizierten Artikel in diesen Zeitschriften innerhalb des analysierten Zeitraums entspricht (120 bzw. $6,75 \%$, wenn das »Journal of Social Issues « nicht einbezogen wird, sondern nur die Mainstream-Zeitschriften). Diese Zahl ist jedoch eine eher konservative Einschätzung, die vermutlich den Anteil friedenspsychologischer Sozialpsychologie unterschätzt, da die analysierten Zeitschriften zum einen allgemein gehalten sind und zum anderen Grundlagenforschung stärker als anwendungsorientierte Forschung vertreten ist. Andere sozialpsychologische Zeitschriften, die entweder inhaltlich spezifischer (wie z. B. »Group Processes and Intergroup Relations«), methodologisch offener und flexibler (wie z. B. »British Journal of Social Psychology«) oder anwendungsorientierter sind (wie z. B. »Journal of Community and Applied Social Psychology «), würden vermutlich einen höheren Anteil sozialpsychologischer Friedensforschung aufweisen. Im Vergleich dazu war fast ein Drittel (32\%) aller Artikel, die in dem analysierten Zeitraum in der friedenspsychologischen Zeitschrift veröffentlicht wurden, sozialpsychologisch ausgerichtet, was die wichtige Rolle der Sozialpsychologie innerhalb der Friedenspsychologie veranschaulicht.

Die Analyse ergab, dass die am häufigsten untersuchten Themen in den ausgewählten Zeitschriften während des untersuchten Zeitraumes (in dieser Reihenfolge) Vorurteile, soziale Identität, Einstellungen gegenüber Fremdgruppen, Intergruppenkontakt, soziale Dominanz, Diskriminierung, rechtsgerichteter Autoritarismus, Rassismus und Bedrohung waren. Die drei am häufigsten verwendeten Theorien waren die soziale Identitätstheorie, die Kontakthypothese und die Theorie sozialer 
Dominanz. Cohrs und Boehnkes (2008) Unterscheidung zwischen Hinderungs- und Begünstigungsfaktoren für negativen oder positiven Frieden zufolge befassen sich die meisten der untersuchten Arbeiten mit Hinderungsfaktoren für positiven Frieden (soziale Dominanz, rechtsgerichteter Autoritarismus, Vorurteile, Diskriminierung, Bedrohung etc.). Das Hauptgewicht liegt also auf struktureller und nicht auf direkter Gewalt. Zum Beispiel handelte über die Hälfte der analysierten Artikel (56\%) von negativen Intergruppenbeziehungen in Abwesenheit direkter Gewalt (z. B. Vorurteile von weißen US-AmerikanerInnen gegenüber AfroamerikanerInnen), während weniger als ein Drittel (31\%) gewaltförmige Konflikte thematisierte (die drei am häufigsten analysierten Konflikte waren dabei, in dieser Reihenfolge, Israel/Palästina, 9/11 und Nordirland). Dieser Schwerpunkt ist unter anderem damit zu erklären, dass die AutorInnen der ausgewählten Artikel sowie Stichproben der berichteten Studien überwiegend aus Nordamerika (55\%) sowie Europa (28\%) und damit aus Regionen stammen, in denen die Bevölkerung in den letzten Jahrzehnten sehr viel weniger von direkter, kollektiver Gewalt auf eigenem Boden betroffen war als von struktureller Gewalt. Außerdem handeln die analysierten Publikationen überwiegend von negativen Prozessen (Hinderungsfaktoren), während positive Begünstigungsfaktoren des Friedens sehr viel weniger thematisiert wurden. Nur $8 \%$ aller Artikel befassten sich mit positivem Frieden.

Andere Schwachstellen der sozialpsychologischen Friedenspsychologie im Mainstream, die sich in dieser Untersuchung ergaben, sind z. B., dass ein Großteil der Stichproben (69\%) studentisch waren, anstatt eine breitere Schicht der Allgemeinbevölkerung zu repräsentieren; dass es relativ wenige Publikationen gab, die sich mit Prävention und Interventionen befassten (19\%) anstatt mit reinem Verstehen und Beschreiben von Zusammenhängen; dass die Methoden überwiegend quantitativ waren (Experimente und Umfragestudien dabei etwa gleich verteilt; qualitative Methoden nur in 15\% der Publikationen vertreten); und dass sich nur ein sehr geringer Anteil (jeweils 5\%) der publizierten Arbeiten mit sozialer Klasse oder mit politischem Aktivismus befasste. Insgesamt ist also die in diesen Zeitschriften vertretene sozialpsychologische Friedenspsychologie methodologisch, thematisch und geographisch recht eingeschränkt, was dem erklärten Sinn der Friedenspsychologie widerspricht (vgl. Christie 2006; Pettigrew 2011).

\subsection{Ergänzungen aus der Befreiungspsychologie}

Diese Blindstellen der (westlich geprägten) sozialpsychologischen Friedensforschung im Mainstream können jedoch durch kritischere Ansätze in der Psychologie ausgeglichen werden, wie z. B. die in Lateinamerika entwickelte Befreiungspsy- 
chologie (Martín-Baró 1994; Montero 2012; Montero/Sonn 2009; Burton/Kagan 2005; Dawes 2001). Die Befreiungspsychologie wurde von dem Sozialpsychologen und Jesuiten-Priester Ignacio Martín-Baró begründet und hat ihren Ursprung in der Befreiungstheologie sowie in einer Kritik an der empirischen, nordamerikanisch geprägten Mainstream-Psychologie (Martín-Baró 1994). Befreiungspsychologie zielt auf die Entwicklung kritischen Bewusstseins (conscientization) ab sowie auf empowerment der Bevölkerung, sich von Unterdrückung und sozial ungerechten Strukturen zu befreien. Forschungsthemen innerhalb der befreiungspsychologischen Tradition sind z. B. Rassismus, soziale Bewegungen und politischer Aktivismus, Demokratisierungsprozesse, Menschenrechte und soziale Gerechtigkeit (siehe Montero/Sonn 2009). Die Befreiungspsychologie konzentriert sich also auf all die Themen, die der oben beschriebenen Inhaltsanalyse zufolge in der sozialpsychologischen Friedensforschung des Mainstreams zu kurz kommen; vor allem auf positive Begünstigungsfaktoren für positiven Frieden, aber auch auf die Erfahrungen von Opfern direkter Gewalt wie Krieg, staatliche Unterdrückung und Genozid (Burton/Kagan 2005). Außerdem arbeiten BefreiungspsychologInnen mit der Allgemeinbevölkerung und mit benachteiligten Gemeinden anstatt mit privilegierten Studierendenstichproben, und sie entwickeln Interventionen, welche die Welt nicht nur zu beschreiben und verstehen, sondern auch zu verändern versuchen (MartínBaró 1994). Ein weiteres Merkmal der Befreiungspsychologie ist, dass sie in vielen, vor allem »nicht-westlichen « Ländern ausgeübt wird, die in der sozialpsychologischen Friedenspsychologie des Mainstreams unterrepräsentiert sind, wie z. B. verschiedene lateinamerikanische Gesellschaften, Südafrika, Mozambique und die Philippinen (Burton/Kagan 2005; Dawes 2001; Montero 2012).

Die Befreiungspsychologie, die auch als eine politische Variante der Sozialpsychologie angesehen werden kann (Montero 2012), ist also eine überaus sinnvolle Ergänzung zur friedenspsychologischen Sozialpsychologie, wie sie im Mainstream zu finden ist. Ihre stärkere Integration würde dazu beitragen, die in der oben vorgestellten Inhaltsanalyse (Vollhardt/Bilali 2008) identifizierten Lücken zu schließen und die Sozialpsychologie für die Friedens- und Konfliktforschung relevanter zu machen. Die methodologischen und meta-theoretischen Kriterien der Befreiungspsychologie entsprechen auch weitgehend denjenigen der Friedenspsychologie (Dawes 2001; Montero/Sonn 2009; Vollhardt/Bilali 2008) sowie anderer alternativer Strömungen innerhalb der Psychologie, wie z. B. der Aktionsforschung (Lewin 1946; siehe auch Brydon-Miller 1997; Maruyama 2008), der Feldforschung (Paluck/Cialdini 2013), der gesellschaftlichen Psychologie (Himmelweit/Gaskell 1990) oder der Gemeindepsychologie (Levine et al. 2004). Beispielsweise ist die Befreiungspsychologie (wie auch die anderen genannten, kritischeren Ausrichtun- 
gen der Psychologie) sehr offen gegenüber verschiedenen Methoden und beschränkt sich nicht nur auf entweder qualitative oder quantitative Forschungstraditionen (Burton/Kagan 2005; siehe auch Groeben/Vollhardt 2012). Es wird ein großes Gewicht auf die Einbeziehung des Kontextes sowie der individuellen und der strukturellen Ebene zugleich gelegt (Martín-Baró 1994). Die Befreiungspsychologie ist außerdem nicht wertneutral, sondern ein »moralisches Projekt» (Burton/Kagan 2005: 69, siehe auch Montero 2012), d. h. eine ethische sowie politisierte Art und Weise, Psychologie zu betreiben, die sich um soziale Gerechtigkeit und Emanzipation von Unterdrückung bemüht (Montero 2012) - mit anderen Worten, um positiven Frieden.

Nicht zuletzt fällt auf, dass in der Befreiungspsychologie einige Grundannahmen und Forschungsthemen zentral verankert sind, die in der Sozialpsychologie und insbesondere in der sozialpsychologischen Friedensforschung in den letzten Jahren langsam Beachtung finden und an Bedeutung gewinnen. Dies umfasst soziale Bewegungen und Aktivismus (Becker 2012; Thomas/Louis 2013; Zomeren et al. 2008); die Rolle von Macht sowie die Notwendigkeit, die Perspektive der Unterdrückten (bzw. von Gruppen mit geringerem sozialen Status) stärker zu berücksichtigen (Dixon et al. 2010; Saguy et al. 2009); und die zentrale Bedeutung der »Wiedererlangung historischer und kollektiver Erinnerungen« (Martín-Baró, 1994: 30), die sich in jüngeren sozialpsychologischen Studien zum kollektiven Gedächtnis und zu historischen Repräsentationen im Allgemeinen (Bilali/Ross 2012; Liu/Hilton 2005; Sibley et al. 2008) sowie nach kollektiver Gewalt wie ethnischen Konflikten und Genozid im Besonderen (Vollhardt/Bilewicz 2013; Wohl/Branscombe 2008) wiederfindet. Es ist also zu hoffen, dass die wichtige Rolle der Befreiungspsychologie (sowie anderer verwandter Strömungen innerhalb der Psychologie) für die sozialpsychologische Friedensforschung zukünftig stärker anerkannt wird und diese Strömungen langfristig besser integriert werden, um eine bedeutungsvollere und einflussreichere sozialpsychologische Friedensforschung zu schaffen.

\section{Neuere Trends in der sozialpsychologischen Friedensforschung}

Im Folgenden werden einige neue Trends der sozialpsychologischen Friedensforschung vorgestellt. Dies erfolgt auf der Grundlage der aktuellsten fünf Jahrgänge (2008-2012) derselben sozialpsychologischen Zeitschriften, auf denen die oben vorgestellte Inhaltsanalyse beruht (mit Ausnahme des »Journal of Social Issues«, das ausschließlich Sonderhefte publiziert). Für diesen aktuellen Überblick wurden die Abstracts aller in Frage kommenden Artikel durchgelesen und die Häufigkeiten der untersuchten Themen, Stichprobenarten und Kontexte der friedenspsycholo- 
gisch relevanten Artikel ausgewertet. Außerdem wurden seltene Methoden vermerkt sowie andere Besonderheiten dieser Arbeiten festgehalten. Diese Analyse ist somit weniger systematisch als die oben vorgestellte Inhaltsanalyse und soll lediglich dazu dienen, einen Überblick über die wichtigsten Trends und Thematiken zu verschaffen, ohne Anspruch auf Vollständigkeit. Viele der genannten Arbeiten sind der »kontextuellen Sozialpsychologie« (Kelman 2012; Pettigrew 1991) verpflichtet.

\subsection{Nachwirkungen kollektiver Gewalt}

Im Hinblick auf Arbeiten über direkte Gewalt fällt auf, dass sozialpsychologische Forschung über die Nachwirkungen von ethnischen Konflikten und Massengewalt (einschließlich Genozid) in den letzten Jahren zugenommen hat (Vollhardt/Bilewicz 2013). Dies ist sicherlich parallel zu ähnlichen Entwicklungen in anderen Disziplinen und interdisziplinären Forschungsbereichen zu sehen, die sich zunehmend mit kollektivem Gedächtnis (Olick et al. 2011), kollektivem Trauma (Alexander et al. 2004; Fassin/Rechtman 2009) sowie Transitional Justice, Wiedergutmachung und Reparationen (z. B. Barkan 2000) beschäftigen. In der Sozialpsychologie besteht dabei ein Fokus auf Prozesse bei Mitgliedern von TäterInnengruppen, insbesondere auf emotionale Reaktionen wie kollektive Schuld und Scham. ${ }^{2}$ Oftmals wird die Auswirkung dieser Emotionen auf die Bereitschaft untersucht, Reparationszahlungen und andere Formen von Wiedergutmachungen zu unterstützen. In manchen Untersuchungen geht es darum, welche psychologischen Prozesse und Interventionen dazu beitragen, dass Mitglieder einer TäterInnengruppe überhaupt die Gewalt anerkennen, die ihre Eigengruppe gegen die Fremdgruppe ausgeübt hat (ČehajićClancy et al. 2011; Gunn/Wilson 2011) bzw. welche Prozesse dieser Anerkennung im Wege stehen (Sibley et al. 2008). Im Allgemeinen ist nämlich das Erleben kollektiver Schuld oder Scham selten (Leach et al. 2013) oder von Dynamiken wie der Schlussstrichdebatte (Imhoff et al. 2013) geprägt. Selbst eine grundsätzliche Anerkennung der Gewalttaten der Eigengruppe ist oft nicht gegeben (Bilali 2013).

Sozialpsychologische Friedensforschung über die Perspektive von Opfergruppen ist seltener. Dennoch hat es auch hier in den vergangenen Jahren einige Vorstöße gegeben, z. B. mit Arbeiten über »competitive victimhood « (Noor et al. 2008), d. h. der Tendenz, in einen Wettbewerb darüber zu treten, welche Gruppe durch den Konflikt am meisten gelitten hat. Diese Forschung hat unter anderem aufgezeigt, dass Personen, die verstärkt um den Opferstatus der Eigengruppe konkurrieren, der anderen Gruppe im Konflikt weniger trauen und weniger Bereitschaft zur Verge-

2 Vgl. hierzu Berndsen/McGarty (2010); Brown/Cehajic (2008); Brown et al. (2008 a); Imhoff et al. (2012); Gunn/Wilson (2011); Peetz et al. (2010); Zagefka et al. (2010). 
bung und Versöhnung zeigen. Grundsätzlicher zeigen experimentelle Studien auf, dass die bloße Erinnerung an das Leiden der Eigengruppe durch einen Genozid oder Krieg dazu führt, dass Gewalthandlungen der Eigengruppe legitimiert werden und weniger kollektive Schuld erlebt wird - selbst dann, wenn es sich um einen unterschiedlichen Kontext mit anderen wahrgenommenen Feindgruppen handelt (Wohl/ Branscombe 2008). Im Gegensatz dazu kann die Erfüllung grundlegender psychologischer Bedürfnisse von Opfergruppen nach empowerment seitens der TäterInnengruppe (wie z. B. Anerkennung der Kompetenzen und Stärken der Opfergruppe) zu erhöhter Versöhnungsbereitschaft führen (Shnabel et al. 2009). Im Allgemeinen hat in den letzten Jahren innerhalb der Sozialpsychologie das Thema der Versöhnung nach Intergruppengewalt an Gewicht gewonnen (Brown et al. $2008 \mathrm{~b}$; Greenaway et al. 2011; Philpot/Hornsey 2008; 2011). Besondere Aufmerksamkeit haben dabei offizielle Entschuldigungen erhalten (Philpot/Hornsey 2008; 2011). Beispielsweise hat diese Forschung untersucht, welche Elemente eine Entschuldigung aufweisen muss, um erfolgreich zu sein (Wohl et al. 2012; siehe auch Wohl et al. 2011) und wie Mitglieder einer TäterInnengruppe auf die Opfergruppe reagieren, wenn die Entschuldigung zurückgewiesen wird (Harth et al. 2011).

Die Forschung zu diesen Themen, die in den Mainstream-Zeitschriften der Sozialpsychologie zu finden ist, befasst sich überwiegend mit individualpsychologischen Prozessen. Es wird dabei meistens nicht berücksichtigt, wie diese Prozesse sozial konstruiert werden oder von gesellschaftlichen Strukturen wie den Machtpositionen der beteiligten Gruppen oder der normativen Unterstützung für Versöhnung beeinflusst werden (für Ausnahmen siehe Paluck 2009; Rimé et al. 2011). Außerdem werden sozialpsychologische Konsequenzen existierender TransitionalJustice-Verfahren nicht explizit untersucht (für eine Ausnahme siehe wieder Rimé et al. 2011). Stattdessen werden überwiegend laborexperimentelle Studien durchgeführt, die oft Texte mit realen Entschuldigungen oder Beschreibungen von historischen Gewalttaten als Untersuchungsmaterialien verwenden. Diese Studien sind außerdem geographisch eingeschränkt. Zwar sind in diesem Forschungsbereich, im Vergleich zu anderen Themen der Sozialpsychologie, relativ diverse Kontexte vertreten (z. B. Bosnien, Chile, Nordirland, Ruanda, Aborigines in Australien), aber diese sind gegenüber Studien in den Vereinigten Staaten oder Westeuropa noch unterrepräsentiert und viele relevante Nachkriegsgesellschaften und aktuelle Konfliktgebiete (wie z. B. Guatemala, Kambodscha, Afghanistan oder Sudan) fehlen bisher ganz. 


\subsection{Einstellungen zu Kriegen und harten Maßnahmen gegen Terrorismus}

Viele aktuelle sozialpsychologische Untersuchungen über Einstellungen zu direkter Gewalt beziehen sich (wie auch schon in dem Zeitraum der oben vorgestellten Inhaltsanalyse) auf den Kontext der Kriege in Afghanistan und Irak seit 2001 sowie auf Maßnahmen gegen Terrorismus in Europa und den Vereinigten Staaten. Bis auf wenige Ausnahmen (z. B. Fisher et al. 2008) untersuchen die Studien dabei aber nur eine Seite, nämlich die Einstellungen der Menschen in den Gesellschaften, die diese Kriege führen. Einstellungen und Konsequenzen bei denjenigen, die angegriffen werden, werden fast nie berichtet.

Mehrere sozialpsychologische Studien haben sich in den letzten Jahren damit befasst, welche psychologischen Prozesse Unterstützung für die Kriege gegen Afghanistan und Irak erklären können. Dabei wurde beispielsweise die Rolle des blinden versus konstruktiven Patriotismus untersucht sowie Attributionen für Ereignisse wie die Terroranschläge am 11. September 2001 (Sahar 2008); der Einfluss von Bindungssicherheit, welche die negative Wirkung von Salienz der eigenen Sterblichkeit auf Unterstützung für den Krieg aufheben kann (Gillath/Harth 2011); und wie Ethnozentrismus die Wertschätzung menschlichen Lebens der Fremd- und Eigengruppe in Kriegen mitbestimmt (Pratto/Glasford 2008). Zusätzlich zu viel erforschten ideologischen Konstrukten wie rechtsgerichteter Autoritarismus und soziale Dominanzorientierung wurde außerdem in vergangenen Jahren untersucht, wie kollektiver Narzissmus (d. h. eine unsichere und defensive soziale Identität, die mit übersteigerter Wertschätzung der Eigengruppe einhergeht) mit Unterstützung für Krieg zusammenhängt (Golec de Zavala et al. 2009). Ähnliche Prozesse und Faktoren wurden auch im Hinblick auf Einstellungen zu harten politischen Maßnahmen gegen Terrorismus untersucht. Hier wurden z. B. Effekte von rechtsgerichtetem Autoritarismus und sozialer Dominanzorientierung (Golec de Zavala/ Kossowska 2011), intrinsischer Religiosität und Sterblichkeitssalienz (Golec de Zavala et al. 2012) sowie dem Bedürfnis nach kognitiver Geschlossenheit (Orehek et al. 2010) festgestellt.

In mehreren Untersuchungen wurden außerdem Einstellungen zu Folter von irakischen Kriegsgefangenen und Zivilisten untersucht. Beispielweise wurden kognitive Strategien erforscht, die es Menschen erlauben, sich von den Gewalttaten der Eigengruppe moralisch zu distanzieren, wie z. B. Dehumanisierung der Opfer (Leidner et al. 2010). Auch wurde aufgezeigt, dass die Wahrnehmung moralischer und sonstiger Überlegenheit der Eigengruppe mit erhöhter Unterstützung für Folter einhergeht (Iyer et al. 2012; Leidner et al. 2010). Außerdem wurden teilweise paradoxe Effekte sowohl von Religiosität (Malka/Soto 2011) als auch von der Salienz 
einer gemeinsamen sozialen Kategorie (»Menschheit«) auf Einstellungen zu Folter im Kontext des Irakkriegs gefunden (Morton/Postmes 2011).

Insgesamt fällt bei diesen Studien auf, dass in den allermeisten Fällen Prozesse und Faktoren untersucht werden, die destruktive Einstellungen zu Krieg (oder deren Abwesenheit) erklären, während Untersuchungen über positive Einstellungen zum Frieden äußerst selten sind. Nur ein Artikel aus den untersuchten Zeitschriften im analysierten Zeitraum beschäftigte sich mit Friedensaktivismus (Simon et al. 2008); lediglich eine weitere Studie untersuchte Einstellungen zu Frieden in einem anderen Zusammenhang, nämlich dem Konflikt zwischen Israel und Palästina (Sharvit et al. 2010). Und Studien zu positiven Einstellungen gegenüber humanitärer Hilfe für Opfer von Krieg und Massenmord wurden nur in drei Artikeln berichtet (Cameron/ Payne 2011; McFarland et al. 2012; Zagefka et al. 2011).

\subsection{Strukturelle Gewalt gegen ethnische Minderheiten}

Sozialpsychologische Friedensforschung beschäftigt sich nach wie vor häufig mit Rassismus und anderen negativen Einstellungen gegenüber ethnischen Minderheiten. Viele der Studien aus den Vereinigten Staaten beziehen sich dabei auf Einstellungen zu AfroamerikanerInnen und (seltener) zu AmerikanerInnen lateinamerikanischer Herkunft. ${ }^{3}$ Untersuchungen aus Europa handeln oft von Einstellungen zu ImmigrantInnen oder Einwanderungsgesetzen (Dhont et al. 2011; Jetten/Wohl 2012; Pereira et al. 2010; Storari/Green 2012) und Forschung aus Australien und Neuseeland thematisiert überwiegend Einstellungen zu Aborigines (Barlow et al. 2012; Sibley et al. 2008). In allen diesen Kontexten werden Einstellungen zu MuslimInnen und Menschen aus dem arabischen Kulturkreis zunehmend häufiger untersucht (Agerström et al. 2012; Lyons et al. 2009; Mange et al. 2012; Smeekes et al. 2012). Studien aus anderen Gesellschaften und Kontinenten (Lateinamerika, Afrika, Asien) sind, wie bereits oben erwähnt, in diesen Zeitschriften stark unterrepräsentiert.

Zudem fällt auf, dass sich diese Forschung überwiegend mit Einstellungen von Mitgliedern der Mehrheitsgesellschaft gegenüber Minderheiten beschäftigt (siehe auch Dixon et al. 2010). Die umgekehrte Perspektive aus Sicht der Minderheitsgruppen ist äußerst selten (Friedman/Saroglou 2010; Martinovic/Verkuyten 2012; Schaafsma 2011; Schaafsma et al. 2010), wie auch Studien über Einstellungen von Minderheitsgruppen zueinander (Barlow et al. 2010; Craig/Richeson 2012).

3 Vgl. hierzu Biernat et al. (2009); Goff et al. (2008); Johnson et al. (2009); Kouchaki (2011); Remedios et al. (2012); Todd et al. (2011). 
Viele der Arbeiten über Faktoren, die zu struktureller Gewalt beitragen, untersuchen kognitive Prozesse der sozialen Kategorisierung und gesellschaftlich übermittelte Stereotype. Außerdem werden häufig individuelle Unterschiede untersucht, z. B. hinsichtlich Perspektivenübernahme, Toleranz, nationaler Identität, dem Bedürfnis nach kognitiver Geschlossenheit, wahrgenommener Bedrohung oder ideologischer Variablen wie sozialer Dominanzorientierung und rechtsgerichtetem Autoritarismus. Viele Studien in diesem Bereich handeln auch von Intergruppenkontakt und den Prozessen, die sich dadurch ändern und positiv auf Einstellungen gegenüber Fremdgruppen auswirken können. Mehrere Arbeiten untersuchen Diskriminierung in der Verteilung von Ressourcen und Jobs (Agerström et al. 2011; Biernat et al. 2009; Kouchaki 2011; Remedios et al. 2011; Yogeswareen/Dasgupta 2012) sowie in anderen Bereichen wie z. B. diskriminierende politische Maßnahmen (Johnson et al. 2009) oder die erhöhte Bereitschaft zu direkter Gewalt gegenüber ethnischen Minderheiten (Goff et al. 2008; Mange et al. 2012). Diese Studien illustrieren also die psychologischen Prozesse, die dazu führen, dass strukturelle Gewalt (nach Galtung 1969) in verminderten Lebenschancen durch eine ungleiche Verteilung von Ressourcen, Bildung und Lebenserwartungen resultieren. Dies ist auch durch mehrere Studien illustriert, welche die Auswirkung von Diskriminierung auf psychologisches und physisches Wohlgefühl, Drogenmissbrauch oder auf die Wahrnehmung von Bildungschancen untersuchen (O'Hara et al. 2012; Schaafsma 2011; Stock et al. 2011).

Wie auch in anderen Bereichen der sozialpsychologischen Friedensforschung sind viele dieser Studien auf individualpsychologische Prozesse konzentriert, ohne strukturelle Faktoren und deren Zusammenwirken mit individuellen Prozessen zu berücksichtigen. Es gibt einige wenige Ausnahmen. Beispielsweise untersuchte eine Arbeit den Einfluss von ethnisch homogenen versus heterogenen Schulen auf Vorurteile und Einstellungen zu Intergruppenkontakt bei Schulkindern (McGlothlin/ Killen 2010). Eine andere Publikation berichtete eine Triangulation von Daten aus experimentellen Studien mit Zeitungsartikeln und Statistiken über Dehumanisierung und Todesurteile für afroamerikanische Gefangene (Goff et al. 2008).

\subsection{Begünstigungsfaktoren positiven Friedens}

Ein deutlicher Trend in der sozialpsychologischen Friedensforschung der letzten Jahre ist die Zunahme an Untersuchungen über gesellschaftliches Engagement. Es werden vor allem psychologische Prozesse und andere Faktoren untersucht, welche die Bereitschaft zu sozialem Protest für soziale Gerechtigkeit und andere konstruktive Ziele beeinflussen. Diese Arbeiten tragen somit zu unserem Wissen über Be- 
günstigungsfaktoren positiven Friedens bei (ein ansonsten eher wenig erforschtes Thema in der Sozialpsychologie). Während sich Forschung in diesem Bereich in den vergangenen Jahren überwiegend darauf konzentrierte, welche psychologischen Prozesse die Beteiligung an Protesten erhöhen (etwa wahrgenommene Ungerechtigkeit und Zorn, kollektive Selbstwirksamkeit sowie eine (politisierte) soziale Identität; siehe z. B. Zomeren et al. 2008), beschreibt die aktuelle Forschung in diesem Bereich Prozesse und Faktoren, die die Protestbereitschaft verringern können. Beispielsweise demonstrieren diese Studien, dass die Protestbereitschaft abnimmt, wenn die von der Eigengruppe erlebte Diskriminierung als legitim wahrgenommen (Jetten et al. 2012) bzw. das System als solches legitimiert wird (Jost et al. 2012). Außerdem wurde herausgefunden, dass benevolenter (zunächst $»$ wohlwollend « erscheinender) Sexismus die Wahrscheinlichkeit herabsetzt, dass Frauen gegen Geschlechterdiskriminierung protestieren, während das bei feindseligem Sexismus nicht der Fall ist (Becker/Wright 2011). Appelle an die "gemeinsame Menschlichkeit« reduzierte außerdem die Bereitschaft bei Mitgliedern historischer Opfergruppen, für Wiedergutmachungen zu protestieren (Greenaway et al. 2011). Unter Mitgliedern der Friedensbewegung in Deutschland wurde herausgefunden, dass eine symbolische Affirmation der Gruppenidentität ebenfalls die Bereitschaft verringerte, weiter zu protestieren (Simon et al. 2008). Diese Untersuchungen demonstrieren also die Effekte verschiedener Beschwichtigungstaktiken, die von Gruppen oder Personen in Machtpositionen zur Unterminierung von Protesten genutzt werden können.

Andere Studien beschäftigten sich mit nicht-normativen Taktiken bei Protesten sowie deren Auswirkungen auf politische und Gruppen-Identifikation (Becker et al. 2011). So wurde beispielsweise aufgezeigt, dass bei nicht-normativen Protesthandlungen andere psychologische Faktoren eine Rolle spielen als bei normativen Protesten, nämlich Verachtung (statt Zorn) und geringe (statt hohe) kollektive Selbstwirksamkeit (Tausch et al. 2011). Zudem wurden Dynamiken und Reaktionen der Polizei auf einen antikapitalistischen Krawall (Cronin/Reicher 2009) untersucht sowie Reaktionen auf Gruppenmitglieder, die gegen Diskriminierung der Eigengruppe protestieren (Jetten et al. 2011).

\section{Sozialpsychologische Friedensforschung im deutschsprachigen Raum}

Da der Großteil der beschriebenen Forschungsarbeiten auf internationaler Ebene angesiedelt ist, stellt sich für ein deutschsprachiges Publikum die Frage: Finden sich ähnliche Ansätze in der hiesigen Forschungslandschaft? 
Im deutschsprachigen Raum besteht mit dem Forum Friedenspsychologie e. V. (http://www.friedenspsychologie.de), das jährlich Fachtagungen durchführt, eine organisatorische Plattform für sozialpsychologische Friedensforschung. Die Tagungstitel der letzten fünf Jahre, »Gruppenbasierte Aggression und Gewalt« (Marburg, 2008), »Attitude Formation, Value Change, and Intercultural Communication« (Bremen, 2009), »Conflict, Peace and Integration« (Bielefeld, 2010), »Social Injustice and Conflict« (Marburg, 2011) und »Täter und Opfer kollektiver Gewalt« (Konstanz, 2012), befinden sich durchaus im Einklang mit den weiter oben aufgezeigten inhaltlichen Trends. Von besonderer Bedeutung für das Vorantreiben der Friedenspsychologie waren in den letzten Jahren insbesondere die Arbeitsgruppe Sozialpsychologie an der Philipps-Universität Marburg mit Forschung zu verschiedenen Aspekten von Intergruppenbeziehungen (Leitung: Ulrich Wagner) und Menschenrechten (federführend: Jost Stellmacher), die sich vor allem mit Medienberichterstattung über Konflikte befassende Projektgruppe Friedensforschung (Leitung: Wilhelm Kempf, ehemals Universität Konstanz), das Team um Klaus Boehnke (Jacobs University Bremen) mit Forschung zu friedenspolitischem Engagement und $\mathrm{zu}$ Intergruppenbeziehungen in der Einwanderungsgesellschaft sowie die Abteilung Kommunikationspsychologie an der Friedrich-Schiller-Universität Jena (Leitung: Wolfgang Frindte) mit Forschung zu Antisemitismus, Terrorismus und Beziehungen zwischen MuslimInnen und Nicht-MuslimInnen in Deutschland (sowie deren mediale Präsenz). Bezüge zur Sozialpsychologie werden für alle diese Forschungsschwerpunkte deutlich, auch wenn von den genannten Personen nur Ulrich Wagner eine Professur für Sozialpsychologie innehat.

Auch außerhalb des Kreises der im Forum Friedenspsychologie Engagierten findet in Deutschland sozialpsychologische Forschung statt, die relevant für die Friedens- und Konfliktforschung ist oder zumindest sein könnte. Dies ist u. a. der Fall für den Lehrstuhl für Sozialpsychologie in Jena, der lange von Amelie Mummendey geführt wurde und den nun Thomas Kessler innehat sowie für den Lehrstuhl für Sozialpsychologie und Politische Psychologie an der Universität Kiel (Bernd Simon) und die Lehrbereiche Sozialpsychologie (Stefan Stürmer) und Community Psychology (Anette Rohmann) an der FernUniversität in Hagen. Mit Forschung zu verschiedenen Aspekten von sozialer Identität und Intergruppenbeziehungen, Kooperation und prosozialem Verhalten, Autoritarismus und politischer Partizipation werden hier wichtige friedenspsychologisch relevante Themen untersucht. Zu nennen wäre weiterhin das Institut für Konflikt- und Gewaltforschung an der Universität Bielefeld, an dem insbesondere der Leiter, Andreas Zick, mit Forschung zu Rechtsextremismus und »gruppenbezogener Menschenfeindlichkeit« (Zick et al. 2008) hervorgehoben werden kann. 
Ein wichtiges, deutschsprachiges Überblickswerk der Friedenspsychologie, in dem auch sozialpsychologische Perspektiven stark vertreten sind, ist der von Sommer und Fuchs (2004) herausgegebene Band »Krieg und Frieden: Handbuch der Konflikt- und Friedensforschung «. Außerdem wurde die Friedenspsychologie in Deutschland exemplarisch in einem Sonderheft der Zeitschrift »Peace and Conflict: Journal of Peace Psychology « (herausgegeben von Boehnke et al. 2005) vorgestellt, mit Beiträgen über Beziehungen zwischen Antisemitismus und sozialer Dominanzorientierung sowie rechtsgerichteten Autoritarismus (Frindte et al. 2005), kognitive Repräsentationen von Menschenrechten (Stellmacher et al. 2005), Werte und Einstellungen zu Krieg (Cohrs et al. 2005), soziale Identität in Zeiten internationalen Konflikts (Kolbe et al. 2005), psychologische Faktoren, welche die Teilnahme an der (deutschen) Friedensbewegung vorhersagen (Boehnke/Boehnke 2005), und über Erfahrungen eines kurdischen AsylbewerberInnen-Paares in Deutschland (Koop 2005). Bis auf den letztgenannten Beitrag sind alle diese Artikel in sozialpsychologischen Theorien und Konstrukten verankert.

\section{Fazit}

In diesem Beitrag wurden einige aktuelle Trends der Forschung im Überschneidungsbereich von Sozialpsychologie und Friedenspsychologie dargestellt sowie einige Schwerpunkte entsprechender Forschung in Deutschland erwähnt. Dass dieses Feld gegenwärtig an Bedeutsamkeit gewinnt, wird auch an einigen kürzlich publizierten Herausgeberbänden deutlich: so das von Linda Tropp (2012) herausgegebene »Oxford Handbook of Intergroup Conflict«, das explizit die Zielsetzung verfolgt, die gegenseitigen Berührungspunkte der Sozial- und der Friedenspsychologie herauszuarbeiten, das von Daniel Bar-Tal (2011) herausgegebene Sammelwerk »Intergroup Conflicts and Their Resolution: A Social Psychological Perspective" sowie die vom selben Autor verfasste Monografie »Intractable Conflict: SocioPsychological Foundations and Dynamics« (2013).

Doch wie kann die interdisziplinäre Friedens- und Konfliktforschung mehr Gebrauch von der Sozialpsychologie machen? Und was kann die Sozialpsychologie im Allgemeinen, zumindest potenziell, zur Friedens- und Konfliktforschung beitragen? Ein wichtiger erster Punkt ist, dass die Sozialpsychologie eine Brückenfunktion einnehmen kann. Da sie sich, zumindest im Sinne der kontextuellen Sozialpsychologie (Pettigrew 1991), mit der Interaktion zwischen Individuen und gesellschaftlichen und institutionellen Faktoren befasst, ist sie eigentlich ideal positioniert, um die »makrowissenschaftlichen « Perspektiven der Soziologie, Politikwissenschaft, Wirtschaftswissenschaften etc. zu ergänzen (Christie et al. 2008; Kel- 
man 2012; Pettigrew 1998). So richtet sie den Blick darauf, wie Individuen Faktoren des gesellschaftlichen Kontextes (z. B. politische Maßnahmen, Ungleichheiten zwischen sozialen Gruppen) interpretieren und mit welchen kognitiven und affektiven Prozessen sie darauf reagieren, die dann entweder zur Legitimierung oder dem Bedürfnis nach Änderung dieser Strukturen beitragen. Sozialpsychologische Konstrukte wie Autoritarismus, soziale Dominanzorientierung, Viktimisierungseinstellungen etc. können somit für die Erklärung behilflich sein, wieso es in bestimmten Kontexten zu konflikteskalierenden oder zu konfliktreduzierenden Reaktionen kommt. Mit ihrem Fokus auf politisches Engagement, Aktivismus und Protestverhalten kann die Sozialpsychologie wiederum zu der Frage beitragen, wie individuelles Verhalten auf den gesellschaftlichen Kontext rückwirken kann (Sherif 1970). Damit haben wir natürlich die Logik des Makro-Mikro-Links nachgezeichnet, wie sie etwa im sogenannten »Badewannenmodell« vertreten wird (Coleman 1991; Esser 1999).

Die genannten Punkte mögen auf der Hand liegen, aber die Beiträge der Sozialpsychologie sind hier nicht trivial. Manchmal werden in den »Makrowissenschaften « implizit (oder auch explizit) psychologische Annahmen gemacht, um Einflüsse von Faktoren des gesellschaftlichen Kontextes zu erklären. Zum Beispiel gehen politologische Analysen im Bereich der internationalen Beziehungen oft davon aus, dass die Motive für Terrorismus rational und mit einer strategischen Kosten-NutzenAnalyse verbunden sind (Pape 2005; siehe auch Ginges et al. 2011), während andere PolitikwissenschaftlerInnen über die Rolle von persönlichen Krisen, kollektivem Trauma oder Identitätskonflikten schreiben (Pedahzur 2005; Roy 2004; siehe auch O’Duffy 2008). Die Validität solcher Annahmen muss aber nachgewiesen werden (Christie et al. 2008; Kelman 2012). Und hierin liegt ein zweiter wichtiger, potenzieller Beitrag der Sozialpsychologie. Mit ihrem breiten Methodenkanon, inklusive der experimentellen Testung theoretisch abgeleiteter Hypothesen (im Labor und im Feld, z. B. Paluck/Cialdini 2013; Paluck/Green 2009), ist sie in der Lage, kausale Annahmen zu prüfen und gegebenenfalls zu verwerfen. Geprüfte und für belastbar befundene Annahmen über relevante psychologische Faktoren können wiederum genutzt werden, um wirkungsvolle Interventionen zu entwickeln.

Ein (seltenes) Beispiel dafür, wie genau dies funktionieren kann und wie SozialpsychologInnen mit internationalen NGOs im Bereich der Friedensarbeit kooperieren können, ist die feldexperimentelle Forschung über die Wirksamkeit von radiobasierten Programmen, die von der NGO Radio La Benevolencija-Humanitarian Tools Foundation produziert werden. Diese Radioprogramme haben zum Ziel, Intergruppenbeziehungen in Nachkriegsgesellschaften in Ruanda, der Demokratischen Republik Kongo und Burundi zu verbessern (Bilali/Vollhardt 2013; Paluck 
2009; 2010; siehe auch Staub et al. 2008). Die Programme basieren auf sozialpsychologischen Theorien über Intergruppenkonflikt und Versöhnung sowie auf klinisch-psychologischen Theorien über Traumatisierung und Traumabewältigung. SozialpsychologInnen arbeiten zusammen mit JournalistInnen daran, diese Prinzipien in Seifenopern einzubetten, die dem lokalen Kontext angepasst sind und die von einem großen Anteil der Bevölkerung (z. B. über 84\% in Ruanda) rezipiert werden. Die Evaluationen dieser Programme werden schließlich von SozialpsychologInnen entworfen und durchgeführt, um Effekte auf Einstellungen, Emotionen, Gruppennormen und Verhalten zu testen und die Programme zu optimieren (Bilali/Vollhardt 2013; Paluck 2009; 2010).

Solche und andere Forschungsbeispiele, die wir in diesem Artikel diskutiert haben, zeigen also das Potenzial der Sozialpsychologie auf, nützliche Beiträge zur interdisziplinären Friedens- und Konfliktforschung zu liefern. Damit dieses Potenzial aber besser genutzt werden kann, muss die sozialpsychologische Friedensforschung sich noch stärker bemühen, über westliche Kontexte hinaus wirklich internationaler zu werden, sich öfter aus dem Labor herauszubewegen, Forschungsfragen in relevante Kontexte einzubetten sowie den Blick für Faktoren und Prozesse auf der Makroebene (inklusive kultureller Einflüsse) nicht zu verlieren - um zu vermeiden, strukturelle Probleme zu entpolitisieren und zu individuellen Problemen zu machen, anstatt sie zu lösen (Martín-Baro 1994). 


\section{Literatur}

Agerström, Jens/Björklund, Fredrik/Carlsson, Rickard/Rooth, Dan-Olof 2012: Warm and Competent Hassan $=$ Cold and Incompetent Eric: A Harsh Equation of Real-Life Hiring Discrimination, in: Basic and Applied Social Psychology 34: 4, 359-366.

Alexander, Jeffrey C./Eyerman, Ron/Giesen, Bernard/Smelser, Neil J./Sztompka, Piotr 2004: Cultural Trauma and Collective Identity, Berkeley, CA.

Allport, Gordon W. 1954: The Historical Background of Modern Social Psychology, in: Handbook of Social Psychology, Reading, MA, 3-56.

Aronson, Elliot/Wilson, Tim/Akert, Robin 2013: Social Psychology, 8. Auflage, Boston, MA.

Barber, Brian K. (Hrsg.) 2009: Adolescents and War: How Youth Deal with Political Violence, New York, NY.

Barkan, Elazar 2000: The Guilt of Nations: Restitution and Negotiating Historical Injustices, Baltimore, MD.

Barlow, Fiona K./Louis, Winnifred R./Terry, Deborah J. 2010: Minority Report: Social Identity, Cognitions of Rejection and Intergroup Anxiety Predicting Prejudice from one Racially Marginalized Group towards Another, in: European Journal of Social Psychology 40: 5, 805-818.

Bar-Tal, Daniel 2004: The Necessity of Observing Real Life Situations: PalestinianIsraeli Violence as a Laboratory for Learning about Social Behaviour, in: European Journal of Social Psychology 34: 6, 677-701.

Becker, Julia C. 2012: The System Stabilizing Role of Identity Management Strategies: Social Creativity Can Undermine Collective Action for Social Change, in: Journal of Personality and Social Psychology 103: 4, 647-662.

Becker, Julia C./Tausch, Nicole/Spears, Russell/Christ, Oliver 2011: Committed Dis(s)idents: Participation in Radical Collective Action Fosters Disidentification with the Broader In-Group but Enhances Political Identification, in: Personality and Social Psychology Bulletin, 37: 8, 1104-1116.

Becker, Julia C./Wright, Stephen C. 2011: Yet Another Dark Side of Chivalry: Benevolent Sexism Undermines and Hostile Sexism Motivates Collective Action for Social Change, in: Journal of Personality and Social Psychology 101: 1, 62-77.

Berndsen, Mariëtte/McGarty, Craig 2010: The Impact of Magnitude of Harm and Perceived Difficulty of Making Reparations on Group-Based Guilt and Reparation towards Victims of Historical Harm, in: European Journal of Social Psychology 40: 3, 500-513. 
Bierhoff, Hans Werner 2006: Sozialpsychologie. Ein Lehrbuch, 6. Auflage, Stuttgart.

Biernat, Monica/Collins, Elizabeth C./Katzarska-Miller, Iva/Thompson, Elizabeth $R$. 2009: Race-Based Shifting Standards and Racial Discrimination, in: Personality and Social Psychology Bulletin 35: 1, 16-28.

Bilali, Rezarta 2013: National Narrative and Social Psychological Influences in the Turkish Denial of the Armenian Genocide, in: Journal of Social Issues 69: 1, 16-33.

Bilali, Rezarta/Ross, Michael A. 2012: Remembering Intergroup Conflict, in: The Oxford Handbook of Intergroup Conflict, New York, NY, 123-135.

Bilali, Rezarta/Vollhardt, Johanna Ray 2013: Priming Effects of a Reconciliation Radio Drama on Historical Perspective Taking in the Aftermath of Mass Violence in Rwanda, in: Journal of Experimental Social Psychology 49: 1, 144-151. Boehnke, Klaus/Boehnke, Mandy 2005: Once a Peacenik - Always a Peacenik? Results from a German Six-Wave, Twenty-Year Longitudinal Study, in: Peace and Conflict: Journal of Peace Psychology 11: 3, 337-354.

Boehnke, Klaus/Fuss, Daniel/Kindervater, Angela 2005: Peace Psychology in Germany, in: Peace and Conflict: Journal of Peace Psychology 11: 3, 229-237.

Bretherton, Diane/Balvin, Nikola (Hrsg.) 2012: Peace Psychology in Australia, New York, NY.

Brown, Rupert/Čehajić, Sabina 2008: Dealing with the Past and Facing the Future: Mediators of the Effects of Collective Guilt and Shame in Bosnia and Herzegovina, in: European Journal of Social Psychology 38: 4, 669-684.

Brown, Rupert/González, Roberto/Zagefka, Hanna/Manzi, Jorge/Čehajić, Sabina 2008 a: Nuestra Culpa: Collective Guilt and Shame as Predictors of Reparation for Historical Wrongdoing, in: Journal of Personality and Social Psychology 94: 1, 75-90.

Brown, Ryan P./Wohl, Michael J.A./Exline, Julie J. 2008 b: Taking Up Offenses: Secondhand Forgiveness and Group Identification, in: Personality and Social Psychology Bulletin 34: 10, 1406-1419.

Brydon-Miller, Mary 1997: Participatory Action Research: Psychology and Social Change, in: Journal of Social Issues 53: 4, 657-666.

Burton, Mark/Kagan, Carolyn 2005: Liberation Social Psychology: Learning from Latin America, in: Journal of Community and Applied Social Psychology 15: 1, 63-78.

Cameron, C. Daryl/Payne, B. Keith 2011: Escaping Affect: How Motivated Emotion Regulation Creates Insensitivity to Mass Suffering, in: Journal of Personality and Social Psychology 100: 1, 1-15. 
Carrier, Martin 2004: Interessen als Erkenntnisgrenzen? Die Wissenschaft unter Verwertungsdruck, in: Hogrebe, Wolfram/Bromand, Joachim (Hrsg.): Grenzen und Grenzüberschreitungen (XIX. Deutscher Kongress für Philosophie), Berlin, 168-180.

Čehajić-Clancy, Sabina/Effron, Daniel A./Halperin, Eran/Liberman, Varda/Ross, Lee D. 2011: Affirmation, Acknowledgment of In-Group Responsibility, GroupBased Guilt, and Support for Reparative Measures, in: Journal of Personality and Social Psychology 101: 2, 256-270.

Christie, Daniel J. 2006: What is Peace Psychology the Psychology of?, in: Journal of Social Issues 62: 1, 1-17.

Christie, Daniel J./Tint, Barbara S./Wagner, Richard V./Winter, Deborah D. 2008: Peace Psychology for a Peaceful World, in: American Psychologist 63: 6, 540-552.

Christie, Daniel J./Wagner, Richard V./Winter, Deborah D. (Hrsg.) 2001: Peace, Conflict, and Violence: Peace Psychology for the 21st Century, Englewood Cliffs, NJ.

Cohrs, J. Christopher/Boehnke, Klaus 2008: Social Psychology and Peace: An Introductory Overview, in: Social Psychology 39: 1, 4-11.

Cohrs, J. Christopher/Moschner, Barbara/Maes, Jurgen/Kielmann, Sven 2005: Personal Values and Attitudes toward War, in: Peace and Conflict: Journal of Peace Psychology 11: 3, 293-312.

Coleman, James S. 1991: Grundlagen der Sozialtheorie. Band 1: Handlungen und Handlungssysteme, München.

Craig, Maureen A./Richeson, Jennifer A. 2012: Coalition or Derogation? How Perceived Discrimination Influences Intraminority Intergroup Relations, in: Journal of Personality and Social Psychology 102: 4, 759-777.

Cronin, Patrick/Reicher, Stephen 2009: Accountability Processes and Group Dynamics: a SIDE Perspective on the Policing of an Anti-Capitalist Riot, in: European Journal of Social Psychology 39: 2, 237-254.

Daiute, Colette 2010: Human Development and Political Violence, Cambridge, MA.

Dawes, Andy 2001: Psychologies for Liberation: Views from Elsewhere in Peace, Conflict, and Violence, in: Christie, Daniel J./Wagner, Richard V./Winter, Deborah (Hrsg.): Peace Psychology for the 21st Century, Englewood Cliffs, NJ, 295-306.

Deutsch, Morton 1995: William James: The First Peace Psychologist, in: Peace and Conflict: Journal of Peace Psychology 1: 1, 27-36. 
Dixon, John/Tropp, Linda R./Durrheim, Kevin/Tredoux, Colin 2010: »Let Them Eat Harmony«: Prejudice-Reduction Strategies and Attitudes of Historically Disadvantaged Groups, in: Current Directions in Psychological Science 19: 2, 76-80.

Dhont, Kristof/Roets, Arne/Hiel, Alain van 2011: Opening Closed Minds: The Combined Effects of Intergroup Contact and Need for Closure on Prejudice, in: Personality and Social Psychology Bulletin 37: 4, 514-528.

Esser, Hartmut 1999: Soziologie. Spezielle Grundlagen. Band 1: Situationslogik und Handeln, Frankfurt a. M.

Fassin, Didier/Rechtman, Richard 2009: The Empire of Trauma: An Inquiry into the Condition of Victimhood, Princeton, NJ.

Friedman, Mike/Saroglou, Vassilis 2010: Religiosity, Psychological Acculturation to the Host Culture, Self-Esteem and Depressive Symptoms among Stigmatized and Nonstigmatized Religious Immigrant Groups in Western Europe, in: Basic and Applied Social Psychology 32: 2, 185-195.

Frindte, Wolfgang/Wettig, Susan/Wammetsberger, Dorit 2005: Old and New AntiSemitic Attitudes in the Context of Authoritarianism and Social Dominance Orientation - Two Studies in Germany, in: Peace and Conflict: Journal of Peace Psychology 11: 3, 239-266.

Fuchs, Albert/Sommer, Gert 2004: Ansatz - Ziele und Aufgaben - Kontroversen in Krieg und Frieden, in: Fuchs, Albert/Sommer, Gert (Hrsg.): Handbuch der Konflikt- und Friedenspsychologie, Weinheim, 3-17.

Galtung, Johan 1969: Violence, Peace and Peace Research, in: Journal of Peace Research 6: 3, 176-191.

Gergen, Kenneth J. 1973: Social Psychology as History, in: Journal of Personality and Social Psychology 26: 2, 309-320.

Gergen, Kenneth J. 2006: Social Psychology and the Wrong Revolution, in: European Journal of Social Psychology 19: 5, 463-484.

Gibson, Stephen 2011: Social Psychology, War and Peace: Towards a Critical Discursive Peace Psychology, in: Social and Personality Psychology Compass 5: 5, 239-250.

Gillath, Omri/Hart, Joshua 2010: The Effects of Psychological Security and Insecurity on Political Attitudes and Leadership Preferences, in: European Journal of Social Psychology 40: 1, 122-134.

Ginges, Jeremy/Atran, Scott/Sachdeva, Sonya/Medin, Douglas 2011: Psychology out of the Laboratory: The Challenge of Violent Extremism, in: American Psychologist 66: 6, 507-519. 
Goff, Phillip A./Eberhardt, Jennifer L./Williams, Melissa J./Jackson, Matthew C. 2008: Not Yet Human: Implicit Knowledge, Historical Dehumanization, and Contemporary Consequences, in: Journal of Personality and Social Psychology 94: 2, 292-306.

Golec de Zavala, Agnieszka/Cichocka, Aleksandra/Eidelson, Roy/Jayawickreme, Nuwan 2009: Collective Narcissism and its Social Consequences, in: Journal of Personality and Social Psychology 97: 6, 1074-1096.

Golec de Zavala, Agnieszka/Cichocka, Aleksandra/Orehek, Edward/Abdollahi, Abdolhossein 2012: Intrinsic Religiosity Reduces Intergroup Hostility under Mortality Salience, in: European Journal of Social Psychology 42: 4, 451-461.

Golec de Zavala, Agnieszka/Kossowska, MaLgorzata 2011: Correspondence between Images of Terrorists and Preferred Approaches to Counterterrorism: The Moderating Role of Ideological Orientations, in: European Journal of Social Psychology 41: 4, 538-549.

Greenaway, Katharine H./Quinn, Emerald A./Louis, Winnifred R. 2011: Appealing to Common Humanity Increases Forgiveness but Reduces Collective Action among Victims of Historical Atrocities, in: European Journal of Social Psychology 41: 5, 569-573.

Greenwood, John D. 2004: The Disappearance of the Social in American Social Psychology, New York, NY.

Groeben, Norbert/Vollhardt, Johanna Ray 2012: Friedenspsychologie als zielorientierte Praxisforschung. Eine metatheoretisch-methodologische Kritik, in: Baros, Wassilios/Rost, Jürgen (Hrsg.): Natur- und kulturwissenschaftliche Perspektiven in der Psychologie. Methodologie - Methoden - Anwendungsbeispiele, Berlin, 17-27.

Gunn, Gregory R./Wilson, Anne E. 2011: Acknowledging the Skeletons in our Closet: The Effect of Group Affirmation on Collective Guilt, Collective Shame, and Reparatory Attitudes, in: Personality and Social Psychology Bulletin 37: 11, 1474-1487.

Hammack, Phillip L. 2010: Identity as Burden or Benefit? Youth, Historical Narrative, and the Legacy of Political Conflict, in: Human Development 53: 4, 173-201.

Harth, Nicole S./Hornsey, Matthew J./Barlow, Fiona K. 2011: Emotional Responses to Rejection of Gestures of Intergroup Reconciliation, in: Personality and Social Psychology Bulletin 37: 6, 815-829.

Heuchert, J. W. P. 2012: Peace Psychology: Contributions from Africa, in: Christie, Daniel J: (Hrsg.): The Encyclopedia of Peace Psychology, Malden, MA, 785-788. 
Himmelweit, Hilde T./Gaskell, George (Hrsg.) 1990: Societal Psychology: Implications and Scope, London.

Hovland, Carl I./Janis, Irving L./Kelley, Harold H. 1953: Communication and Persuasion: Psychological Studies of Opinion Change, New Haven, CT.

Imhoff, Roland/Bilewicz, Michael/Erb, Hans-Peter 2012: Collective Regret versus Collective Guilt: Different Emotional Reactions to Historical Atrocities, in: European Journal of Social Psychology 42: 6, 729-742.

Imhoff, Roland/Wohl, Michael J. A./Erb, Hans-Peter 2013: When the Past is Far from Dead: How Ongoing Consequences of Genocides Committed by the Ingroup Impact Collective Guilt, in: Journal of Social Issues 69: 1, 74-91.

Iyer, Aarti/Jetten, Jolanda/Haslam, S. Alexander 2012, Sugaring o'er the Devil: Moral Superiority and Group Identification Help Individuals Downplay the Implications of Ingroup Rule-Breaking, in: European Journal of Social Psychology 42: 2, 141-149.

James, William 1995: The Moral Equivalent of War, in: Peace and Conflict: Journal of Peace Psychology 1: 1, 17-26.

Jetten, Jolanda/Schmitt, Michael T./Branscombe, Nyla R./Garza, Azenett A./Mewse, Avril J. 2011: Group Commitment in the Face of Discrimination: The Role of Legitimacy Appraisals, in: European Journal of Social Psychology 41: 1, 116-126.

Jetten, Jolanda/Wohl, Michael J. A. 2012: The Past as a Determinant of the Present: Historical Continuity, Collective Angst, and Opposition to Immigration, in: European Journal of Social Psychology 42: 4, 442-450.

Johnson, James D./Olivo, Nelgy/Gibson, Nathan/Reed, William/Ashburn-Nardo, Leslie 2009: Priming Media Stereotypes Reduces Support for Social Welfare Policies: The Mediating Role of Empathy, in: Personality and Social Psychology Bulletin 35: 4, 463-476.

Jost, John T./Chaikalis-Petritsis, Vagelis/Abrams, Dominic/Sidanius, Jim/Toorn, Jojaneeke van der,/Bratt, Christopher 2012: Why Men (and Women) Do and Don't Rebel: Effects of System Justification on Willingness to Protest, in: Personality and Social Psychology Bulletin 38: 2, 197-208.

Kaminer, Debra/Eagle, Gillian/Stevens, Garth/Higson-Smith, Craig (Hrsg.) 2013: Special Issue: Continuous Traumatic Stress, in: Peace and Conflict: Journal of Peace Psychology 19: 2.

Kelman, Herbert C. (Hrsg.) 1965: International Behavior: A Social Psychological Analysis, New York, NY. 
Kelman, Herbert C. 2012: Social Psychology and the Study of Peace: Personal Reflections, in: Tropp, Linda (Hrsg.): The Oxford Handbook of Intergroup Conflict, New York, NY, 361-372.

Kolbe, Michaela/Boos, Margarete/Gurtner Andrea 2005: Social Identity in Times of International Conflict, in: Peace and Conflict: Journal of Peace Psychology 11: 3, 313-336.

Koop, Ingrid I. 2005: Refugees in Church Asylum: Intervention between Political Conflict and Individual Suffering, in: Peace and Conflict: Journal of Peace Psychology 11: 3, 355-365.

Kouchaki, Maryam 2011: Vicarious Moral Licensing: The Influence of Others Past Moral Actions on Moral Behavior, in: Journal of Personality and Social Psychology 101: 4, 702-715.

Leach, Colin W./Bou Zeinnedine, Fouad/Cehajic-Clancy, Sabina 2013: Moral Immemorial: The Rarity of Self-Criticism for Previous Generations Genocide or Mass Violence, in: Journal of Social Issues 69: 1, 34-53.

Leidner, Bernhard/Castano, Emanuele/Zaiser, Erica/Giner-Sorolla, Roger 2010: Ingroup Glorification, Moral Disengagement, and Justice in the Context of Collective Violence, in: Personality and Social Psychology Bulletin 36: 8, 1115-1129.

Levin, Shana/Henry, P. J./Pratto, Felicia/Sidanius, Jim 2003: Social Dominance and Social Identity in Lebanon: Implications for Support of Violence against the West, in: Group Processes/Intergroup Relations 6: 4, 353-368.

Levine, Murray/Perkins, Douglas D./Perkins, David V. 2004: Principles of Community Psychology: Perspectives and Applications, New York, NY.

Lewin, Kurt 1946: Action Research and Minority Problems, in: Journal of Social Issues 2: 4, 34-46.

Liu, James H./Hilton, Denis J. 2005: How the Past Weighs on the Present: Social Representations of History and Their Role in Identity Politics, in: British Journal of Social Psychology 44: 4, 537-556.

Lyons, Patricia A./Kenworthy, Jared B./Popan, Jason R. 2010: Ingroup Identification and Group-Level Narcissism as Predictors of U.S. Citizens Attitudes and Behavior toward Arab Immigrants, in: Personality and Social Psychology Bulletin 36: 9, 1267-1280.

Malka, Ariel/Soto, Christopher J. 2011: The Conflicting Influences of Religiosity on Attitude toward Torture, in: Personality and Social Psychology Bulletin 37: 8, 1091-1103. 
Mange, Jessica/Chun, Woo Y./Sharvit, Keren/Belanger, Jocelyn J. 2012: Thinking about Arabs and Muslims Makes Americans Shoot Faster: Effects of Category Accessibility on Aggressive Responses in a Shooter Paradigm, in: European Journal of Social Psychology 42: 5, 552-556.

Martín-Baró, Ignacio 1994: Writings for a Liberation Psychology, Cambridge, MA.

Martinovic, Borja/Verkuyten, Maykel 2012: Host National and Religious Identification among Turkish Muslims in Western Europe: The Role of Ingroup Norms, Perceived Discrimination and Value Incompatibility, in: European Journal of Social Psychology 42: 7, 893-903.

Maruyama, Geoffrey M. 2008: Program Evaluation, Action Research, and Social Psychology: A Powerful Blend for Addressing Applied Problems, in: Sansone, Carol/Morf, Carolyn C./Panter, A. T. (Hrsg.): The Sage Handbook of Methods in Social Psychology, Thousand Oaks, CA, 429-442.

McFarland, Sam/Webb, Matthew/Brown, Derek 2012: All Humanity Is My Ingroup: A Measure and Studies of Identification with All Humanity, in: Journal of Personality and Social Psychology 103: 5, 830-853.

McGlothlin, Heidi/Killen, Melanie 2010: How Social Experience Is Related to Children's Intergroup Attitudes, in: European Journal of Social Psychology 40: 4, 625-634.

Milgram, Stanley 1974: Obedience to Authority, New York, NY.

Moghaddam, Fathalie M./Marsella, Anthony J. (Hrsg.) 2003: Understanding Terrorism: Psychosocial Roots, Consequences and Interventions, Washington, D.C. Montero, Maritza 2012: Liberation Psychology, in: Christie, Daniel J. (Hrsg.): The Encyclopedia of Peace Psychology, Malden, MA, 599-603.

Montero, Maritza/Sonn, Christopher C. (Hrsg.) 2009: Psychology of Liberation: Theory and Applications, New York, NY.

Montiel, Cristina J./Noor, Noraini M. (Hrsg.) 2009: Peace Psychology in Asia, New York, NY.

Morton, Thomas A./Postmes, Tom 2011: What Does it Mean to be Human? How Salience of the Human Category Affects Responses to Intergroup Harm, in: European Journal of Social Psychology 41: 7, 866-873.

O'Duffy, Brendan 2008: Radical Atmosphere: Explaining Jihadist Radicalization in the UK, in: Political Science and Politics 41: 1, 37-42.

O'Hara, Ross E./Gibbons, Frederick X./Weng, Chih-Yuan/Gerrard, Meg/Simons, Ronald L. 2012: Perceived Racial Discrimination as a Barrier to College Enrollment for African Americans, in: Personality and Social Psychology Bulletin 38: $1,77-89$. 
Olick, Jeffrey/Vinitzky-Seroussi, Vered/Levy, Daniel (Hrsg.) 2011: The Collective Memory Reader, New York, NY.

Orehek, Edward/Fishman, Shira/Dechesne, Mark/Doosje, Bertjan/Kruglanski, Arie/Cole, Angela P./Saddler, Billie/Jackson, Tarra 2010: Need for Closure and the Social Response to Terrorism, in: Basic and Applied Social Psychology 32: 4, 279-290.

Paluck, Elizabeth L. 2009: Reducing Intergroup Prejudice and Conflict Using the Media: A Field Experiment in Rwanda, in: Journal of Personality and Social Psychology 96: 3, 574-587.

Paluck, Elizabeth L. 2010: Is it Better Not to Talk? Group Polarization, Extended Contact, and Perspective-Taking in Eastern Democratic Republic of Congo, in: Personality and Social Psychology Bulletin 36: 9, 1170-1185.

Paluck, Elizabeth L./Cialdini, Robert B. 2013: Field Research Methods, in: Reis, Harry T./Judd, Charles M. (Hrsg.): Handbook of Research Methods in Personality and Social Psychology, Cambridge, MA, i.E.

Paluck, Elizabeth L./Green, Donald P. 2009: Prejudice Reduction: What Works? A Review and Assessment of Research and Practice, in: Annual Review of Psychology 60, 339-367.

Pape, Robert A. 2005: Dying to Win: The Strategic Logic of Suicide Terrorism, New York, NY.

Peetz, Johanna/Gunn, Gregory/Wilson, Anne E. 2010: Crimes of the Past: Defensive Temporal Distancing in the Face of Past In-Group Wrongdoing, in: Personality and Social Psychology Bulletin 36: 5, 598-611.

Pedahzur, Ami 2005: Suicide Terrorism, Cambridge, MA.

Pereira, Cícero/Vala, Jorge/Costa-Lopes, Rui 2010: From Prejudice to Discrimination: The Legitimizing Role of Perceived Threat in Discrimination against Immigrants, in: European Journal of Social Psychology 40: 7, 1231-1250.

Pettigrew, Thomas F. 1991: Toward Unity and Bold Theory: Popperian Suggestions for Two Persistent Problems of Social Psychology, in: Stephan, Cookie W./ Stephan, Walter G./Pettigrew, Thomas F. (Hrsg.): The Future of Social Psychology: Defining the Relationship Between Sociology and Psychology, New York, NY, 13-27.

Pettigrew, Thomas F. 1998: Applying Social Psychology to International Social Issues, in: Journal of Social Issues 54: 4, 663-675.

Pettigrew, Thomas F. 2006: The Advantages of Multilevel Approaches, in: Journal of Social Issues 62: 3, 615-620.

Pettigrew, Thomas F. 2011: Toward Sustainable Psychological Interventions for Change, in: Peace and Conflict: Journal of Peace Psychology 17: 2, 179-192. 
Philpot, Catherine R./Hornsey, Matthew J. 2008: What Happens when Groups Say Sorry: The Effect of Intergroup Apologies on Their Recipients, in: Personality and Social Psychology Bulletin 34: 4, 474-487.

Philpot, Catherine R./Hornsey, Matthew J. 2011: Memory for Intergroup Apologies and its Relationship with Forgiveness, in: European Journal of Social Psychology 41: 1, 96-106.

Pratto, Felicia/Glasford, Demis E. 2008: Ethnocentrism and the Value of a Human Life, in: Journal of Personality and Social Psychology 95: 6, 1411-1428.

Pyszczynski, Thomas A./Solomon, Sheldon/Greenberg, Jeff 2003: In the Wake of 9/11: The Psychology of Terror, Washington, D.C.

Remedios, Jessica D./Chasteen, Alison L./Oey, Elvina 2012: »Unskilled« Workers: Social Skills Stereotypes Affect Evaluations of Biracial Job Applicants, in: Basic and Applied Social Psychology 34: 3, 204-211.

Rimé, Bernard/Kanyangara, Patrick/Yzerbyt, Vincent/Paez, Dario 2011: The Impact of Gacaca Tribunals in Rwanda: Psychosocial Effects of Participation in a Truth and Reconciliation Process after a Genocide, in: European Journal of Social Psychology 41: 1, 695-706.

Roy, Olivier 2004: Globalised Islam: The Search for a New Umma, London.

Saguy, Tamar/Tausch, Nicole/Dovidio, John F.,/Pratto, Felicia 2009: The Irony of Harmony: Intergroup Contact Can Produce False Expectations for Equality, in: Psychological Science 20: 1, 114-121.

Sahar, Gail 2008: Patriotism, Attributions for the 9/11 Attacks, and Support for War: Then and Now, in: Basic and Applied Social Psychology 30: 2, 189-197. Schaafsma, Juliette 2011: Discrimination and Subjective Well-Being: The Moderating Roles of Identification with the Heritage Group and the Host Majority Group, in: European Journal of Social Psychology 41: 6, 786-795.

Schaafsma, Juliette/Nezlek, John B./Krejtz, Izabela/Safron, Magdalena 2010: Ethnocultural Identification and Naturally Occurring Interethnic Social Interactions: Muslim Minorities in Europe, in: European Journal of Social Psychology 40: 6, 1010-1028.

Sharvit, Keren/Bar-Tal, Daniel/Raviv, Amiram/Raviv, Alona/Gurevich, Ronit 2010: Ideological Orientation and Social Context as Moderators of the Effect of Terrorism: The Case of Israeli-Jewish Public Opinion Regarding Peace, in: European Journal of Social Psychology 40: 1, 105-121.

Sherif, Muzafer 1970: On the Relevance of Social Psychology, in: American Psychologist 25: 2, 144-156. 
Shnabel, Nurit/Nadler, Arie/Ullrich, Johannes/Dovidio, John F./Carmi, Dganit 2009: Promoting Reconciliation through the Satisfaction of the Emotional Needs of Victimized and Perpetrating Group Members: The Needs-Based Model of Reconciliation, in: Personality and Social Psychology Bulletin 35: 8, 1021-1030. Sibley, Chris G/Liu, James H./Duckitt, John/Khan, Sammyh S. 2008: Social Representations of History and the Legitimation of Social Inequality: The Form and Function of Historical Negation, European Journal of Psychology 38: 3, 542-565.

Simon, Bernd/Trötschel, Roman/Dähne, Dorit 2008: Identity Affirmation and Social Movement Support, in: European Journal of Social Psychology 38: 6, 935-946.

Smeekes, Anouk/Verkuyten, Maykel/Poppe, Edwin 2012: How a Tolerant Past Affects the Present: Historical Tolerance and the Acceptance of Muslim Expressive Rights, in: Personality and Social Psychology Bulletin 38: 11, 1410-1422.

Sommer, Gert/Fuchs, Albert (Hrsg.) 2004: Krieg und Frieden. Handbuch der Konflikt- und Friedenspsychologie, Weinheim.

Staub, Ervin/Pearlman, Laurie A./Bilali, Rezarta 2008: Psychological Recovery, Reconciliation and the Prevention of New Violence: An Approach and its Uses in Rwanda, in: Hart, Barry (Hrsg.): Peacebuilding in Traumatized Societies, Washington, D.C., 131-154.

Stellmacher, Jost/Sommer, Gert/Brahler, Elmar 2005: The Cognitive Representation of Human Rights: Knowledge, Importance, and Commitment, in: Peace and Conflict: Journal of Peace Psychology 11: 3, 267-292.

Stock, Michelle L./Gibbons, Frederick X./Walsh, Laura A./Gerrard, Meg 2011: Racial Identification, Racial Discrimination, and Substance Use Vulnerability among African American Young Adults, in: Personality and Social Psychology Bulletin, 37: 10, 1349-1361.

Storari, Chiara C./Green, Eva G. T. 2012: Intergroup Distinctiveness and Discriminatory Immigration Attitudes: The Role of National Identification, in: Basic and Applied Social Psychology 34: 4, 367-375.

Tausch, Nicole/Becker, Julia C./Spears, Russell/Christ, Oliver/Saab, Rim/Singh, Purnima/Siddiqui, Roomana N. 2011: Explaining Radical Group Behavior: Developing Emotion and Efficacy Routes to Normative and Nonnormative Collective Action, in: Journal of Personality and Social Psychology 101: 1, 129-148. Thomas, Emma F./Louis, Winnifred R. 2013: Doing Democracy: The Social Psychological Mobilization and Consequences of Collective Action, in: Social Issues and Policy Review 7: 1, 173-200. 
Todd, Andrew R./Bodenhausen, Galen V./Richeson, Jennifer A./Galinsky, Adam

D. 2011: Perspective Taking Combats Automatic Expressions of Racial Bias, in: Journal of Personality and Social Psychology 100: 6, 1027-1042.

Vollhardt, Johanna Ray/Bilali, Rezarta 2008: Social Psychology's Contribution to the Psychological Study of Peace: A review, in: Social Psychology 39: 1, 12-25. Vollhardt, Johanna Ray/Bilewicz, Michal 2013: After the Genocide: Psychological

Perspectives on Victim, Bystander, and Perpetrator Groups, in: Journal of Social Issues 69: 1, 1-15.

Wessells, Michael 2006: Child Soldiers: From Violence to Prevention, Cambridge, MA.

Wohl, Michael J. A./Branscombe, Nyla R. 2008: Remembering Historical Victimization: Collective Guilt for Current Ingroup Transgressions, in: Journal of Personality and Social Psychology 94: 6, 988-1006.

Wohl, Michael J. A./Hornsey, Matthew J.,/Bennett, Shannon H. 2012: Why Group Apologies Succeed and Fail: Intergroup Forgiveness and the Role of Primary and Secondary Emotions, in: Journal of Personality and Social Psychology 102: 2, 306-322.

Wohl, Michael J. A./Hornsey, Matthew J./Philpot, Catherine R. 2011: A Critical Review of Official Public Apologies: Aims, Pitfalls, and a Staircase Model of Effectiveness, in: Social Issues and Policy Review 5: 1, 70-100.

Yogeeswaran, Kumar/Dasgupta, Nilanjana 2010: Will the »Real« American Please Stand Up? The Effect of Implicit National Prototypes on Discriminatory Behavior and Judgments, in: Personality and Social Psychology Bulletin 36: 10, 1332-1345.

Zagefka, Hanna/Noor, Masi/Brown, Rupert/Moura, Georgina R. de/Hopthrow, Tim 2011: Donating to Disaster Victims: Responses to Natural and Humanly Caused Events, in: European Journal of Social Psychology 41: 3, 353-363.

Zagefka, Hanna/Pehrson, Samuel/Mole, Richard C. M./Chan, Eva 2010: The Effect of Essentialism in Settings of Historic Intergroup Atrocities, in: European Journal of Social Psychology 40: 5, 718-732.

Zick, Andreas/Wolf, Carina/Küpper, Beate/Davidov, Eldad/Schmidt, Peter/Heitmeyer, Wilhelm 2008: The Syndrome of Group-Focused Enmity: The Interrelation of Prejudices Tested with Multiple Cross-Sectional and Panel Data, in: Journal of Social Issues 64: 2, 363-383.

Zomeren, Martijn van/Postmes, Tom/Spears, Russell 2008: Toward an Integrative Social Identity Model of Collective Action: A Quantitative Research Synthesis of Three Socio-Psychological Perspectives, in: Psychological Bulletin 134: 4, 504-535. 


\section{Die AutorInnen}

Dr. Johanna Ray Vollhardt ist Assistant Professor of Psychology an der Clark University, Worcester, USA.

E-Mail: JVollhardt@clarku.edu

Dr. J. Christopher Cohrs ist Assistant Professor of Psychology an der Jacobs University Bremen, School of Humanities and Social Sciences.

E-Mail: c.cohrs@jacobs-university.de 